\title{
DESAFIOS NA CONSTRUÇÃO DE UMA REDE DE ATENÇÃO EM SAÚDE MENTAL*
}

Luciane Ramos Silva ${ }^{1}$, Carmem Lúcia Colomé Beck², Mariangela Gobatto ${ }^{3}$, Caliandra Marta Dissen ${ }^{4}$, Rosângela Marion da Silva ${ }^{5}$, Natiellen Quatrin Freitas ${ }^{6}$

RESUMO: Esta pesquisa do tipo estudo de caso e de abordagem qualitativa teve como objetivo apresentar e discutir os desafios encontrados para estruturar uma Rede de Atenção em Saúde Mental em um município do Rio Grande do Sul, Brasil. Os dados foram coletados por meio de análise documental, observações não-participantes e grupo focal. A análise foi realizada com base na análise de conteúdo. Dos resultados emergiram os eixos temáticos Entendimento duvidoso sobre a rede por parte dos componentes da Comissão de Saúde Mental; O trabalho identificado como distinto do trabalho real em Saúde Mental; $O$ distanciamento do nivel de atenção terciária com a rede (internação hospitalar) e $A$ falta de proximidade entre as instituições formadoras e os serviços de saúde mental do município. Evidenciou-se neste estudo a necessidade de investir no desenvolvimento dos trabalhadores de saúde para o trabalho em rede, sobretudo na Enfermagem, para expandí-lo na organização da saúde mental. PALAVRAS-CHAVE: Saúde mental; Psiquiatria comunitária; Redes comunitárias; Assistência à saúde.

\section{CHALLENGES IN THE CONSTRUCTION OF A MENTAL HEALTH CARE NETWORK}

ABSTRACT: This research, of the case study type and with a quantitative approach, aimed to present and discuss the challenges met in setting up a Mental Health Care Network in a municipality of Rio Grande do Sul. The data was collected through analysis of documents, non-participant observation, and focus groups. Analysis was undertaken based on content analysis. The following thematic axes emerged from the results: Dubious understanding about the network on the part of the members of the Mental Health Commission; The work identified as distinct from the work currently done in mental health; The distancing between the tertiary level of care and the network (hospitalization) and The lack of proximity between the training institutions and the municipality's mental health services. The study evidenced the need for investment in the development of health workers for work in networks, above all in Nursing, to extend it in the organization of mental health. KEYWORDS: Mental health; Community psychiatry; Community networks; Health care.

\section{DESAFÍOS EN LA CONSTRUCCIÓN DE UNA RED DE ATENCIÓN EN SALUD MENTAL}

RESUMEN: Esta investigación del tipo estudio de caso y de abordaje cualitativo tuvo como objetivo presentar y discutir los desafíos para estructurar una Red de Atención en Salud Mental en un Município de Rio Grande do Sul, Brasil. Los datos fueron obtenidos por medio del análisis documental, observaciones no participantes y grupo focal. El análisis fue realizado con base en el análisis de contenido. De los resultados, surgieron los ejes temáticos Entendimiento sobre la red por los componentes de la Comisión de Salud Mental; El trabajo identificado como distinto del trabajo real en salud mental; El alejamiento del nível de atención terciaria con la red (internación hospitalar) y La falta de proximidad entre las instituciones formadoras y los servicios de salud mental del municipio. En este estudio, se volvió evidente la necesidad de invertir en el desarrollo de los trabajadores de salud para el trabajo en red, principalmente en la enfermería, a fin de expandirlo en la organización de la salud mental.

PALABRAS CLAVE: Salud mental; Psiquiatría comunitaria; Redes comunitarias; Asistência a la salud.

\footnotetext{
*Artigo referente à dissertação intitulada - Entre Fios e Dobras: o tecer da Reforma Psiquiátrica no município de Santa Maria/RS, apresentada em 2009 ao Programa de Pós-Graduação em Enfermagem da Universidade Federal de Santa Maria - PPGEnf UFSM.

${ }^{1}$ Enfermeira. Mestre em Enfermagem. Membro do Grupo de Pesquisa - Trabalho, Saúde, Educação e Enfermagem da UFSM.

${ }^{2}$ Enfermeira. Doutora em Enfermagem. Professora do Departamento em Enfermagem da UFSM. Membro do Grupo de Pesquisa - Trabalho, Saúde, Educação e Enfermagem. Coordenadora da linha de pesquisa Saúde do Trabalhador.

${ }^{3}$ Enfermeira. Mestranda em Enfermagem pelo PPGEnfUFSM. Membro do Grupo de Pesquisa - Trabalho, Saúde, Educação e Enfermagem. ${ }^{4}$ Acadêmica de Enfermagem pela UFSM. Membro do Grupo de Pesquisa Trabalho, Saúde, Educação e Enfermagem. Bolsista PIBIC/CNPq. ${ }^{5}$ Enfermeira do Hospital Universitário de Santa Maria. Doutoranda em Enfermagem DINTER UNIFESP-UFRJ/EEAN-UFSM. Membro do Grupo de Pesquisa - Trabalho, Saúde, Educação e Enfermagem.

${ }^{6}$ Acadêmica de Enfermagem pela UFSM. Membro do Grupo de Pesquisa - Trabalho, Saúde, Educação e Enfermagem. Bolsista PROBIC/ FAPERGS.
} 


\section{INTRODUÇÃO}

A Reforma Psiquiátrica no Brasil surge em favor da mudança dos modelos de atenção e gestão nas práticas de saúde mental ${ }^{(1)}$. Nessa linha, procura-se modificar o sistema de tratamento clínico aos usuários portadores de transtornos mentais, eliminando gradualmente a internação como único modo de tratamento e acrescentando à terapêutica uma rede de serviços territoriais de atenção psicossocial ${ }^{(2)}$.

No entanto, essa dinâmica exige participação competente dos trabalhadores, associada a uma cultura de rede no campo da saúde. A implementação das redes de atenção à saúde mental pode oferecer, na prática, a possibilidade de uma atenção integral, buscando contemplar as múltiplas necessidades do usuário e de sua família, em uma sequência de ações que funcionem articuladas para possibilitar o cuidado integral ao portador de transtorno mental ${ }^{(3)}$.

Frente a essas reflexões emergentes no âmbito da atenção à saúde mental de maneira global e por compreender a complexidade do processo da Reforma Psiquiátrica para muito além da estruturação de serviços substitutivos, foi constituída uma Comissão de Saúde Mental (CSM) em um município localizado no Estado do Rio Grande do Sul/Brasil. A CSM tinha o intuito de contribuir na efetivação da Reforma Psiquiátrica no âmbito municipal por meio de reuniões semanais compostas por trabalhadores dos serviços de saúde mental e de outros serviços de atenção à saúde do município, docentes e discentes de cursos da saúde. A partir das reuniões, emergiu a necessidade de que fosse construída uma rede de atenção em saúde mental que tivesse o papel facilitador na complementaridade do atendimento aos portadores de transtornos mentais, fazendo cumprir os preceitos da Reforma Psiquiátrica.

Sabe-se que a construção de uma rede de serviços de saúde é uma atividade complexa, pois é formada por uma série de pontos de encontro, de trajetórias de cooperação, de simultaneidade de iniciativas e de atores sociais envolvidos ${ }^{(4)}$. No Sistema Único de Saúde, a rede de atenção à saúde mental define-se como de base comunitária, sendo fundamento para a construção desta rede a presença de um movimento permanente e direcionado para os outros espaços da cidade, em busca da emancipação das pessoas com transtornos mentais $^{(5)}$, aspecto fundamental para a consolidação da Reforma Psiquiátrica. Nesse contexto este estudo justifica-se pela necessidade de construir novos saberes em saúde mental, na direção da consolidação da rede de atenção a saúde mental e da Reforma Psiquiátrica. A pesquisa tem por objetivo apresentar e discutir os desafios encontrados para estruturar uma Rede de Atenção em Saúde Mental.

\section{MÉTODO}

Trata-se de um estudo com abordagem qualitativa do tipo Estudo de Caso, sendo que esta escolha se justifica pelo fato de que este propõe desenvolver uma investigação empírica que focaliza um fenômeno dentro do contexto da vida real, especialmente quando os limites entre o fenômeno e o contexto não estão claramente definidos ${ }^{(6)}$.

A pesquisa foi desenvolvida junto a Comissão de Saúde Mental (CSM) de um município do Rio Grande do Sul/Brasil, localizado na região central do estado mencionado, que conta em sua Rede de Atenção Básica à Saúde com 31 serviços (18 Unidades Básicas de Saúde e 13 Estratégias de Saúde da Família), distribuídos por regiões sanitárias. Participou do estudo a totalidade dos integrantes da CSM, ou seja, 11 participantes. Para a coleta dos dados foram utilizadas a análise documental, observações não-participantes e grupo focal.

Com relação aos documentos, foram analisadas as atas da CSM do período de agosto de 2006 a setembro de 2008, as quais foram disponibilizadas pelo Coordenador da Política de Saúde Mental do município. As observações não-participantes foram realizadas pelo pesquisador e um auxiliar de pesquisa e uso de um roteiro e diários de campo para os registros, os quais auxiliaram na captura dos dados uma vez que as manifestações dos participantes ocorriam de maneira verbal e não verbal. As observações foram previamente combinadas com o grupo e ocorreram no período de março a abril de 2009 durante as reuniões da CSM, com duração aproximada de duas horas.

O grupo focal foi constituído pelos integrantes da comissão, sendo realizados em dois momentos no ano de 2009 e contaram com a participação de oito e seis participantes, respectivamente. O material coletado foi analisado com base na análise de conteúdo ${ }^{(7)}$. Para o desencadeamento do processo de análise foram utilizadas as seguintes etapas: pré-análise; descrição analítica e escolha dos temas.

Os participantes deste estudo foram identificados pela letra A-Ator, seguida de número arábico relativo à ordem de manifestação dos participantes. Foram cumpridos os preceitos da Resolução 196/96 do Conselho Nacional de Pesquisa ${ }^{(8)}$ e os participantes foram 
orientados a ler o Termo de Consentimento Livre e Esclarecido e assinaram o mesmo. Este estudo recebeu aprovação do Comitê de Ética em Pesquisa da Universidade Federal de Santa Maria sob o número do Certificado de Apresentação para Apreciação Ética 0283.0.243.000-08, Processo n. 23081.019090/2008-11.

\section{RESULTADOS}

Participaram do estudo os trabalhadores dos serviços de saúde mental, ou seja, coordenadores da Política Municipal de Saúde Mental e do ambulatório de Saúde Mental, e trabalhadores dos Centros de Atenção Psicossocial, da região sanitária Norte e de outros serviços de atenção à saúde, e o diretor da Atenção Básica. Somaram-se a esses, atores formadores (docentes) e profissionais em formação dos cursos de graduação em Enfermagem e Psicologia (discentes), os quais eram conhecedores da realidade enfrentada e discutida do referido município.

A partir dos dados coletados foram construídos os eixos temáticos: Entendimento duvidoso sobre a rede por parte dos componentes da Comissão da Saúde Mental; O trabalho prescrito foi identificado como distinto do trabalho real em Saúde Mental; O distanciamento do nível de atenção terciária para com a rede (internação hospitalar) e A falta de proximidade entre as instituições formadoras e os serviços de saúde mental do município.

\section{DISCUSSÃO}

Com base na análise das atas das reuniões da CSM dos anos de 2007 e 2008, a construção da rede de atenção em saúde mental do referido município foi iniciada com a elaboração dos fluxogramas. O termo fluxograma designa uma representação gráfica de um determinado processo ou fluxo de trabalho que permite demonstrar o caminho ou percurso percorrido pelo mesmo ${ }^{(9)}$. Desta forma, pretendia-se padronizar o sistema de referência e de contra-referência em saúde mental. Nesse contexto, elencou-se o primeiro desafio deste processo que foi o entendimento duvidoso sobre a rede por parte dos componentes da CSM, conforme relatos decorrentes da realização dos grupos focais:

[...] os dispositivos da Política Nacional de Humanização devem se fazer presentes não só na CSM mas, principalmente, nos serviços de atenção psicossocial pensados como estratégicos para a organização da rede de atenção em saúde mental, por manter nossas demandas aquecidas. (A2)
[...] considerando as necessidades dos nossos usuários, temos que considerar caso a caso, não estou falando só dos planos terapêuticos, mas de pensar a organização dos serviços como redes quentes, permeada pelo vínculo, pela responsabilização. (A5)

Percebe-se, analisando os fragmentos acima, que há dificuldade por parte dos integrantes da comissão em relacionar e diferenciar o conceito de rede na teoria com a aplicação na prática. Há a defesa da construção de redes quentes que consistem na conjugação geradora de efeitos de diferenciação, as quais estão presentes na Política Nacional de Humanização ${ }^{(10)}$. No entanto, a execução deste processo resultou em uma rede linear e fria, ou seja, generalizada e não focada no vínculo e na responsabilização, o que destaca a falta de entendimento do que sejam redes.

Nesse sentido, pode-se inferir que a limitação na continuidade do cuidado a portadores de transtornos mentais nos serviços de saúde não está associada a atributos individuais dos pacientes, mas sim a fatores de contexto, incluindo a (des)organização do sistema de saúde do município ${ }^{(11)}$. Assim, é necessário que o significado, bem como o funcionamento da rede esteja claro no modo de pensar e de agir de cada trabalhador para que, de fato, o usuário portador de transtornos mentais tenha suas demandas atendidas de modo integral e satisfatório e para que não seja prejudicada a continuidade do cuidado para esses sujeitos.

Com base na análise das observações das reuniões da CSM, ao avaliar os fluxogramas da rede de atenção do município identificou-se que, além da dúvida em relação à concepção de rede, o trabalho prescrito é distinto do trabalho real em Saúde Mental o que, cotidianamente, dificulta a estruturação de uma rede, já que não se sabe com referência a qual trabalho os fluxogramas devem estar baseados e, posteriormente, executados.

Sabe-se da necessidade de se ter o trabalho prescrito, pois a organização é necessária para toda atividade humana, porém não é adequado restringir os trabalhadores ao trabalho prescrito de forma que ele interfira, ou até mesmo impeça a sua realização ${ }^{(12)}$. Logo, compartilha-se a ideia de que cada situação de trabalho é peculiar e exige estratégias de execução elaboradas pelos trabalhadores, que conforme sua margem de autonomia, experiências, habilidades e competências conseguirão ser mais ou menos eficazes no controle dos efeitos da discrepância entre o prescrito e o real ${ }^{(13)}$.

Apesar dos desafios, foram construídos fluxogramas lineares que atendiam as necessidades dos serviços de 
saúde mental, bem como ao propósito de redes e da continuidade da assistência. Deste modo, compreende-se que mudanças devam ocorrer no sentido de fortalecer o trabalho de saúde mental em rede, havendo implicação do próprio trabalhador, mais do que das forças externas. Assim, ir além do prescrito é fundamental neste processo, sendo indispensável a autonomia e o conhecimento do trabalhador de saúde mental ${ }^{(12)}$.

Outro desafio encontrado na construção da Rede de Atenção à Saúde Mental desse município foram as tensões entre os diferentes níveis de atenção, especificamente, o distanciamento do nível de atenção terciária da rede (internação hospitalar). A fala a seguir exemplifica essa situação:

[...] sob o meu ponto de vista, é nosso papel sim promover esta integração principalmente com os CAPS [Centro de Atenção Psicossocial]. Não estamos aqui para reproduzir a lógica manicomial. Bem pelo contrário, devemos nos unir para fazer valer os pressupostos da Reforma Psiquiátrica. (A10)

Esse fato também foi identificado no registro das atas e do diário de campo, pois as ações desenvolvidas vinculavam-se às iniciativas individuais dos trabalhadores de uma unidade de internação hospitalar psiquiátrica que acreditavam na integração dos Centros de Atenção Psicossocial com as unidades de internação hospitalares, mesmo sem haver um comprometimento institucional da unidade hospitalar. Em outra fala, percebe-se a indignação de um desses trabalhadores com essa situação, mesmo após a construção dos fluxogramas que deveriam auxiliar para que fatos assim não acontecessem.

O paciente fica solto [...] caso tu não estivesses lá, nós não conseguiríamos internar, mesmo naquela situação. (A9)

A articulação entre os serviços de saúde hospitalares e o Centro de Atenção Psicossocial pode caracterizá-lo como espaço social de produção de sujeitos sociais e de subjetividades, espaços de convivência, de sociabilidade, de solidariedade e de inclusão ${ }^{(14)}$. Nesse contexto, percebe-se ainda a supremacia do hospital sobre a rede básica de atenção, figurando como principal local de busca dos usuários que depositam nesse ambiente, segurança e garantia de acolhimento para o portador de sofrimento mental.

Por mais que se amplie e se aprimore a rede básica, as pessoas continuam buscando as emergências e o hospital, talvez como uma estratégia intuitiva e selvagem na busca da integralidade ${ }^{(15)}$. No entanto, a aproximação do serviço de atenção da rede primária com a rede terciária, ou seja, dos Centros de Atenção Psicossocial com as unidades de internação, configura-se como estratégia para tornar vigente $\mathrm{o}$ atual modelo de atenção à saúde mental para aqueles usuários que ainda mantém o vínculo com a instituição hospitalar. Contudo, esse distanciamento do hospital afeta a rede de atenção como um todo, trazendo à tona o sentimento de desamparo por parte dos usuários e dos seus familiares.

Assim, há a necessidade da integração e cooperação entre os serviços de saúde existentes, buscando a promoção de vida comunitária e de autonomia dos usuários, articulando demais recursos, quando necessários, em setores, como jurídicos, econômicos, laborais, empresariais, entre outros, fazendo valer a complexidade das relações humanas ${ }^{(16)}$.

Outro desafio foi a falta de proximidade entre as instituições formadoras e os serviços de saúde mental do município. Apesar de haver a possibilidade de convênios e parcerias com um hospital de ensino, isso não se concretizou. É possível que várias questões interfiram neste processo, ou seja, a falta de preparo dos docentes dos cursos da área da saúde na área de saúde mental, bem como dos trabalhadores hospitalares, a ausência destas discussões nos projetos político-pedagógicos, dentre outros. Os registros no diário de campo igualmente evidenciaram esse distanciamento, conforme manifestações de membros da CSM:

[...] isso já vem de muito tempo, o municipio desencadeou esse processo de buscar uma aproximação, muitos encontros aconteceram, mas, até então, tem sido uma via de mão única. (A2)

\section{[...] quando se fala em aproximação, essa é no sentido de uma relação reciproca. (A7)}

Frente ao exposto, aponta-se para a realidade de que a formação profissional está mais focada nos serviços tecnificados, baseado no modelo biomédico e no processo saúde-doença, em que o hospital está no topo de uma hierarquia qualitativa de trabalho. Neste cenário, por vezes, os interesses corporativos e particulares dos pares pode prevalecer e prejudicar as transformações necessárias nesta área ${ }^{(17)}$. Depreende-se daí uma das barreiras para o avanço das mudanças preconizadas pelo SUS, dentre as quais, a da Reforma Psiquiátrica, considerando a forma- 
ção deficiente dos trabalhadores da saúde para enfrentar o novo modelo de atenção à saúde mental ${ }^{(12)}$. É necessário que os atores envolvidos nas ações em saúde mental aprofundem seus conhecimentos e marcos conceituais acerca das práticas em saúde mental e direcionem suas ações, qualificando sua prática profissional ${ }^{(18)}$.

Percebe-se que algumas instituições formadoras de trabalhadores de saúde se eximem do processo político e social da saúde voltado à implantação de uma rede integrada, de base comunitária, com ofertas complexas de atenção médico-social, sob a diretriz da reabilitação psicossocial. Ao mesmo tempo, concorda-se que um hospital de ensino vinculado a uma instituição formadora deve pertencer à rede de serviços de saúde como uma estratégia complementar na rede de cuidados representada pelo sistema de saúde, cumprindo papéis de apoio matricial, avaliação de tecnologias, desenvolvimento de estratégias assistenciais e métodos de tratamento que possam ser, gradativamente, incorporados pelos serviços de alta resolutividade ambulatorial e acompanhamento domiciliar ${ }^{(19)}$.

Nessa perspectiva, ressalta-se a importância e a responsabilidade de uma instituição pública de ensino, não só pela formação dos profissionais com o perfil para atuar junto ao Sistema Único de Saúde, como também pela assistência atribuída a essa instituição como integrante da rede de saúde do município.

Destaca-se que prestar assistência de saúde para pessoas em sofrimento é um ato de busca e resgate da integralidade do sujeito ${ }^{(20)}$. Contudo, defende-se que a qualidade e a continuidade do cuidado dependem da articulação entre atenção básica e saúde mental em seus diferentes níveis de complexidade. Articulação esta desde o local mais próximo do usuário, passando pelos serviços substitutivos e envolvendo os hospitais de referência quando necessário, contribuindo para a melhoria da assistência prestada e ampliação do acesso da população aos serviços.

\section{CONSIDERAÇÕES FINAIS}

Este estudo identificou como desafios encontrados para estruturar uma Rede de Atenção em Saúde Mental o entendimento duvidoso sobre a rede por parte dos componentes da Comissão de Saúde Mental; o trabalho prescrito identificado como distinto do trabalho real em Saúde Mental; o distanciamento do nível de atenção terciária com a rede (internação hospitalar) e a falta de proximidade entre as instituições formadoras e os serviços de saúde mental do município.
Muitos são os desafios encontrados na construção de uma Rede de Atenção em Saúde Mental nos municípios e o mesmo pode ser melhor enfrentado se houver associação de esforços entre os serviços de saúde e as instituições formadoras de trabalhadores da saúde.

Logo, este estudo contribui no sentido de estimular discussões sobre a complexidade da estruturação de um serviço de rede. Neste sentido, julga-se essencial investir no desenvolvimento de habilidades dos trabalhadores da saúde para o trabalho voltado para essa dinâmica, a fim de expandi-lo na organização da atenção, focada na estrutura da rede básica e nos processos de hierarquização por encaminhamentos e devolução à porta de entrada.

\section{REFERÊNCIAS}

1. Ministério da Saúde (BR). Reforma psiquiátrica e política de saúde mental no Brasil. Secretaria de Atenção à Saúde. DAPE. Coordenação Geral de Saúde Mental. Documento apresentado à Conferência Regional de Reforma dos Serviços de Saúde Mental: 15 anos depois de Caracas. OPAS. Brasília; 2005. [acesso em 28 fev 2012]. Disponível: http://bvsms.saude.gov.br/ bvs/publicacoes/Relatorio15_anos_Caracas.pdf

2. Ministério da Saúde (BR). Saúde mental no SUS: os centros de atenção psicossocial/ Ministério da Saúde. Secretaria de Atenção à Saúde, Departamento de Ações Programáticas Estratégicas. - Brasília; 2004. [acesso em 25 mar 2012]. Disponível: http://www.ccs.saude. gov.br/saude_mental/pdf/SM_Sus.pdf

3. Silva SF. Redes de atenção á saúde no SUS: o pacto pela saúde e redes regionalizadas de ações de serviços de saúde. Campinas, SP: IDISA: CONASEMS; 2008. [acesso em 10 jan 2012]. Disponível: http://observasaude.fundap.sp.gov. br/pacto/Redesatencao/Paginas/Default.aspx

4. Amarante PD. Saúde mental e atenção psicossocial. Temas em saúde. Rio de Janeiro: Fiocruz; 2007.

5. Mello MF, Mello AAF, Kohn R. Epidemiologia da saúde mental no Brasil. Porto Alegre: Artmed; 2007.

6. Yin RK. Estudo de caso: planejamento e métodos. Trad. de Daniel Grassi. 3ª ed. Porto Alegre: Bookman; 2006.

7. Bardin L. Análise de conteúdo. $7^{\mathrm{a}}$ ed. Portugal: Geográfica; 2009.

8. Ministério da Saúde(BR). Conselho Nacional de Saúde. Normas para pesquisa envolvendo seres humanos. Resolução, n. 196/96. Brasília, DF; 1996. 
9. Nunes P. Conceito de fluxograma. 2009. [acesso em 20 mai 2012] Disponível: http:/www.knoow.net/ cienceconempr/gestao/fluxograma.htm\#vermais.

10. Fagundes SMS. Águas da pedagogia da implicação: intercessões da educação para políticas públicas de saúde [dissertação]. Porto Alegre(RS): Universidade Federal do Rio Grande do Sul; 2006.

11. Oliveira GL, Caiaffa WT, Cherchiglia ML. Saúde mental e a continuidade do cuidado em centros de saúde de Belo Horizonte, MG. Rev SaúdePública [Internet]2008;42(4) [acesso em 28 fev 2012]. Disponível: http://www.scielosp.org/scielo. php?script $=$ sci_arttext\&pid=S0034-89102008000400018\&ln $\mathrm{g}=$ en\&nrm=iso $>$. Epub July 03, 2008. ISSN 0034-8910. http:// dx.doi.org/10.1590/S0034-89102008005000038

12. Ramos LS. Entre Fios e Dobras: o tecer da reforma psiquiátrica no Município de Santa Maria/RS [dissertação]. Santa Maria (RS): Universidade Federal de Santa Maria; 2009.

13. Trierweiller AC, Azevedo BM, Pereira VLDV, Cruz RM, Gontijo LA, Santos Júnior, RLF. A estratégia operatória utilizada pelos trabalhadores e o hiato existente entre o trabalho prescrito e o trabalho real. Gestão Industrial. 2008;4(1):101-15.

14. Souza AC. Ampliando o campo da atenção psicossocial: a articulação dos Centros de Atenção Psicossocial com a Saúde da Família. Esc Anna Nery. 2006;10(4):703-10.

15. Cecílio LCO, Merhy E. Integralidade do cuidado como eixo da gestão hospitalar. Campinas: arquivo eletrônico; 2003. [acesso em 10 fev 2012] Disponível: www.hc.ufmg.br/gids/anexos/Integralidade.pdf

16. Schneider ARS. A rede de atenção em saúde mental: a importância da interação entre a atenção primária e os serviços de saúde mental. Cienc Saúde. 2009;2(2):78-84.

17. Ceccim RB, Ferla AA. Educação e saúde: ensino e cidadania como travessia de fronteiras. Trab. Educ. Saúde. 2009;6(3):443-56.

18. Nascimento AAMN, Braga VAB. Atenção em saúde men $\neg$ tal: a prática do enfermeiro e do médico de Programa Saúde da Família de Caucaia-CE. Cogitare enferm. [Internet] 2004;9(1) [acesso em 20 abr 2012]. Disponível: http://ojs.c3sl.ufpr.br/ojs2/index.php/ cogitare/article/viewFile/1709/1417.

19. Carvalho YM, Ceccim RB. Formação e educação em saúde: aprendizados com a saúde coletiva. In: Minayo MCS, Carvalho YM, Campos GWS, Drumond Júnior
M, Akerman M. Tratado de saúde coletiva. $2^{\mathrm{a}}$ ed. São Paulo: Hucitec; Rio de Janeiro: Fiocruz; 2009. p. 137-70

20. Ribeiro C, Ribeiro L, Oliveira A. A construção da assistência à saúde mental em duas Unidades de Saúde da Família de Cuiabá-MT. Cogitare enferm. [Internet] 2008;13(4) [acesso em 10 mai 2012]. Disponível: http://ojs.c3sl.ufpr.br/ojs2/index.php/cogitare/article/ view/13115/8873

Cogitare Enferm. 2012 Out/Dez; 17(4):649-54 